\title{
Polynomial Root-Finding Methods Whose Basins of Attraction Approximate Voronoi Diagram
}

\author{
Bahman Kalantari
}

Received: 5 January 2010 / Revised: 19 December 2010 / Accepted: 5 January 2011 /

Published online: 18 February 2011

(C) Springer Science+Business Media, LLC 2011

\begin{abstract}
Given a complex polynomial $p(z)$ with at least three distinct roots, we first prove that no rational iteration function exists where the basin of attraction of a root coincides with its Voronoi cell. In spite of this negative result, we prove that the Voronoi diagram of the roots can be well approximated through a high order sequence of iteration functions, the Basic Family, $B_{m}(z), m \geq 2$. Let $\theta$ be a simple root of $p(z), V(\theta)$ its Voronoi cell, and $A_{m}(\theta)$ its basin of attraction with respect to $B_{m}(z)$. We prove that given any closed subset $C$ of $V(\theta)$, including any homothetic copy of $V(\theta)$, there exists $m_{0}$ such that for all $m \geq m_{0}, C$ is also a subset of $A_{m}(\theta)$. This implies that when all roots of $p(z)$ are simple, the basins of attraction of $B_{m}(z)$ uniformly approximate the Voronoi diagram of the roots to within any prescribed tolerance. Equivalently, the Julia set of $B_{m}(z)$, and hence the chaotic behavior of its iterations, will uniformly lie to within prescribed strip neighborhood of the boundary of the Voronoi diagram. In a sense, this is the strongest property a rational iteration function can exhibit for polynomials. Next, we use the results to define and prove an infinite layering within each Voronoi cell of a given set of points, whether known implicitly as roots of a polynomial equation, or explicitly via their coordinates. We discuss potential application of our layering in computational geometry.
\end{abstract}

Keywords Complex polynomials · Voronoi diagram $\cdot$ Zeros $\cdot$ Newton's method · Iteration functions · Fractal · Julia set · Dynamical systems · Computational geometry

B. Kalantari $(\bowtie)$

Department of Computer Science, Rutgers University, Newark, USA

e-mail: kalantari@cs.rutgers.edu 


\section{Introduction}

Consider a complex polynomial

$$
p(z)=a_{n} z^{n}+\cdots+a_{1} z+a_{0}
$$

of degree $n \geq 1$. Let the roots of $p(z)$ be $\left\{\theta_{1}, \ldots, \theta_{n}\right\}$. The Voronoi cell of a particular root $\theta$, denoted by $V(\theta)$, is the set of all points in the complex plane that are closer to $\theta$ than to any of the other roots. Each Voronoi cell is an open set with polygonal boundary, possibly unbounded, see Fig. 1. Voronoi cells of distinct roots together with their boundaries partition the plane into disjoint sets. The subject of Voronoi diagram and their applications are well studied in the literature, see, e.g., [7, 16], and the survey [2].

Newton's method for solving the polynomial equation $p(z)=0$ consists of the fixed point iteration:

$$
z_{k+1}=\mathbf{N}\left(z_{k}\right)=z_{k}-\frac{p\left(z_{k}\right)}{p^{\prime}\left(z_{k}\right)}, \quad k=0,1, \ldots,
$$

where $z_{0}$ is a starting complex number. The sequence $\left\{z_{k}\right\}_{k=0}^{\infty}$ is called the orbit of $z_{0}$. The basin of attraction of a root $\theta$ is the set of all points whose orbits under the iterations of Newton's method converge to $\theta$. We will denote this by $A(\theta)$. It is an open set and a part of the so-called Fatou set of $\mathbf{N}(z)=z-p(z) / p^{\prime}(z)$. The basin of attraction of each root consists of the union of connected components, called Fatou components. The immediate basin of attraction of a root is the connected component that contains the root. From the local convergence behavior of Newton's method, the immediate basin of attraction of a root is well-defined. The boundary of the Fatou components forms the Julia set, known to exhibit fractal behavior for general polynomials. For precise definition of Fatou and Julia sets with respect to general rational functions and their properties, see, e.g., [3, 10, 15].

Cayley in [4] studied the behavior of Newton's method in the complex plane for solving the equation $z^{n}-1=0$. In the case of the quadratic equation $z^{2}-1=0$, it is easy to prove that the basin of attraction of each root coincides with its Voronoi cell. The connection between Voronoi cells and basins of attraction of roots of unity fails to be valid for $n \geq 3$. While this can formally be proved, even for $n=3$, the visualization of the shape of the basins of attraction of the roots under Newton's had to wait for many more years, in particular the advent of computers, before it was revealed to have fractal boundary. Fractal images corresponding to Newton's method applied to the equation for cubic roots of unity are well-known and have been reproduced numerous times. While either visually or analytically it is easy to conclude that the basins of attraction of the roots of $z^{3}-1$ under Newton's method are not identical with their Voronoi cells, the basins of attraction do, in fact, resemble a rough approximation to Voronoi diagram of the roots. The same remarks apply to general polynomials, as well as rational iteration functions other than Newton's. However, in general, the quality of this approximation depends on the underlying polynomial, the region through which one may consider the Voronoi cell of a particular root, as well as other parameters, including, of course, the underlying iteration function. 
Fig. 1 The Voronoi cell of a root $\theta$ and its homothetic shrinkage centered at $\theta$

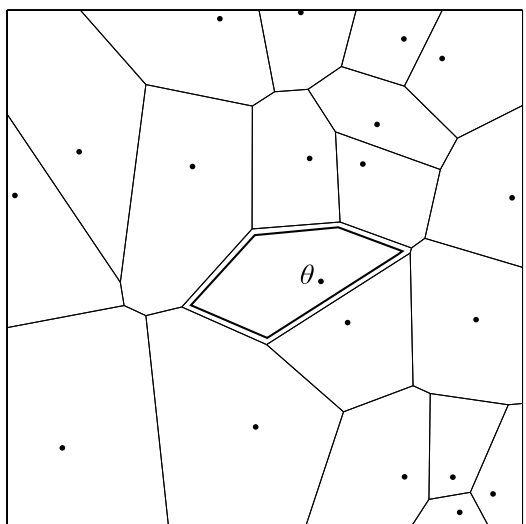

McMullen [14] has shown that there is no generally convergent rational iteration function for polynomial root-finding and this, in particular, implies that given any rational iteration function one can find polynomials for which the particular iteration function would not converge to a root on a set of positive measure. Despite this, given a particular root $\theta$ of a particular polynomial $p(z)$ and a particular iteration function, it is natural to ask how well the basin of attraction $A(\theta)$ may approximate the Voronoi cell $V(\theta)$.

In this article, we prove two results which can be summarized as follows. First, we prove that for a complex polynomial with three or more distinct roots, no rational iteration function can enjoy the property that the basin of attraction of a root would coincide with its Voronoi cell. Second, given an arbitrary polynomial, by making use of a fundamental family of iteration functions, the Basic Family $\left\{B_{m}(z), m \geq 2\right\}$, we prove that its members do asymptotically give a very good approximation of the Voronoi cells to within any homothetic shrinkage of the Voronoi cells centered at the roots. While the first result is a negative result, the second result, our main result, establishes a strong connection between polynomial root-finding and Voronoi cells, while proving a powerful and novel property of the Basic Family. For a study of many properties of this family and their applications, see [10].

\section{Impossibility of Rational Iterations with Voronoi Cell as Basin of Attraction}

Here we prove a negative result in terms of the approximation of Voronoi cell of a root via rational iteration functions defined next.

Definition 2.1 Given a complex polynomial $p(z)$, a rational map

$$
R(z)=\frac{P(z)}{Q(z)},
$$

where $P(z)$ and $Q(z)$ are complex polynomials assumed to be relatively prime, is said to be an iteration function for $p(z)$ if each $\operatorname{root} \theta$ of $p(z)$ is an attractive fixed 
point of $R(z)$, i.e.,

$$
R(\theta)=\theta, \quad\left|R^{\prime}(\theta)\right|<1
$$

For such an iteration function, the fixed point iteration

$$
z_{k+1}=R\left(z_{k}\right), \quad k=0,1, \ldots
$$

is locally convergent for each root of $p(z)$, given an appropriate seed $z_{0}$. Analogous to the case of Newton's function, the notion of an orbit, basin of attraction, Julia and Fatou sets can be defined with respect to iterations of $R(z)$, see, e.g., [3, 10, 15].

The degree of $R(z)$ is the maximum of the degrees of $P(z)$ and $Q(z)$. It is well known that a rational map has either $0,1,2$, or infinitely many Fatou components (see, e.g., [3], Theorem 5.6.2). If $p(z)$ has at least three distinct roots, then there are at least three Fatou components. It thus follows that no rational iteration function for $p(z)$ could result in Fatou components that would coincide with the Voronoi cells of the roots of $p(z)$. The following result, however, proves the impossibility of the coincidence of even one Voronoi cell of a root $\theta$, and its corresponding basin of attraction.

Theorem 2.2 Let $R(z)$ be a rational iteration function for a polynomial $p(z)$ having at least three distinct roots. Given a root $\theta$ of $p(z)$, let $A(\theta)$ be the basin of attraction of $\theta$ with respect to the fixed point iterations of $R(z)$, and $V(\theta)$ the Voronoi cell of $\theta$ with respect to the roots of $p(z)$. Then $A(\theta)$ cannot coincide with $V(\theta)$.

Proof Assume $V(\theta)=A(\theta)$. It is well known that the boundary of $A(\theta)$ coincides with the Julia set of $R$, denoted as $J(R)$. Furthermore, $J(R)$ coincides with the basin of attraction of any other root $\theta^{\prime}$ of $p(z)$, see, e.g., $[3,10,15]$. Thus to prove the theorem, it suffices to show that the boundary of $V(\theta)$ does not coincide with the boundary of other roots of $p(z)$.

The boundary of $V(\theta)$ consists of line segments, or half-lines, or lines. Consider a line segment on the boundary of $V(\theta)$, see Fig. 2. Take an arbitrary point $u$ on this line segment and consider the intersection of a small neighborhood of $u$ and the complement of $V(\theta)$, a semisphere. Since, by assumption, there are at least two other roots $\theta^{\prime}$ and $\theta^{\prime \prime}$, this semisphere must necessarily contain points $v$ and $w$ belonging to the basin of attraction of $\theta^{\prime}$ and $\theta^{\prime \prime}$. Consider the line segment connecting $v$ and $w$. By the least upper-bound property of the reals, this line segment must contain a boundary point of the basins $A\left(\theta^{\prime}\right)$ and $A\left(\theta^{\prime \prime}\right)$. In particular, this implies that there is a boundary point not belonging to the boundary of $V(\theta)$, a contradiction.

Remark 2.3 It is likely that the theorem remains valid if instead of requiring the coincidence of $V(\theta)$ and the basin attraction of $\theta$, we ask if $V(\theta)$ could be a Fatou component of the basin attraction, i.e., only one of the components of the basin of attraction. Regardless of the validity of this, next we prove a strong connection between the Voronoi diagram of polynomial roots and the basins of attraction of its roots via the Basic Family. 
Fig. 2 A neighborhood of a boundary point $u$ of $V(\theta)$ containing two points from the basin of attraction of two other roots

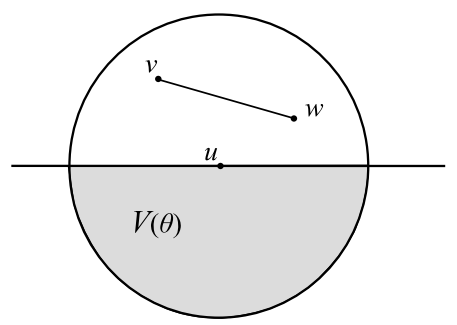

\section{Basic Family of Iteration Functions}

In this section, we describe the Basic Family of iteration functions for polynomial root-finding and summarize their relevant properties. Let $p(z)$ be a given complex polynomial of degree $n$.

Set $D_{0}(z)=1$, and for each $m \geq 2$ define the $m \times m$ matrix determinant

$$
D_{m}(z)=\operatorname{det}\left(\begin{array}{ccccc}
p^{\prime}(z) & \frac{p^{\prime \prime}(z)}{2 !} & \ldots & \frac{p^{(m-1)}(z)}{(m-1) !} & \frac{p^{(m)}(z)}{(m) !} \\
p(z) & p^{\prime}(z) & \ddots & \ddots & \frac{p^{(m-1)}(z)}{(m-1) !} \\
0 & p(z) & \ddots & \ddots & \vdots \\
\vdots & \vdots & \ddots & \ddots & \frac{p^{\prime \prime}(z)}{2 !} \\
0 & 0 & \ldots & p(z) & p^{\prime}(z)
\end{array}\right)
$$

The Basic Family then is the collection of iteration functions

$$
B_{m}(z)=z-p(z) \frac{D_{m-2}(z)}{D_{m-1}(z)}, \quad m=2,3, \ldots
$$

Specific members, $B_{2}(z)$ and $B_{3}(z)$, are the Newton and Halley iteration functions, respectively. These two by themselves posses a rich and interesting history, see [18], also [10]. The Basic Family was studied by Schröder [17], see also [10, 18] for several different but equivalent formulations, as well as many other properties.

The following theorem summarizes some of the main properties of the Basic Family, those relevant to what is to be proved. The theorem can be viewed as a particular case of a determinantal Taylor's theorem.

Theorem $3.1[8,10]$ Let $p(z)$ be a complex polynomial of degree $n$, and $\theta$ a root. For each $m \geq 2$, the following expansion is valid

$$
B_{m}(z)=z-p(z) \frac{D_{m-2}(z)}{D_{m-1}(z)}=\theta+(-1)^{m} \sum_{k=m}^{m+n-2} \frac{\widehat{D}_{m-1, k}(z)}{D_{m-1}(z)}(\theta-z)^{k},
$$


where for each $m \geq 1$ and each $k=m+1, \ldots, m+n-1$,

$$
\widehat{D}_{m, k}(z)=\operatorname{det}\left(\begin{array}{ccccc}
\frac{p^{\prime \prime}(z)}{2 !} & \frac{p^{\prime \prime \prime}(z)}{3 !} & \cdots & \frac{p^{(m)}(z)}{(m) !} & \frac{p^{(k)}(z)}{k !} \\
p^{\prime}(z) & \frac{p^{\prime \prime}(z)}{2 !} & \ddots & \frac{p^{(m-1)}(z)}{(m-1) !} & \frac{p^{(k-1)}(z)}{(k-1) !} \\
p(z) & p^{\prime}(z) & \ddots & \vdots & \vdots \\
\vdots & \vdots & \ddots & \frac{p^{\prime \prime}(z)}{2 !} & \frac{p^{(k-m+2)}(z)}{(k-m+2) !} \\
0 & 0 & \ldots & p^{\prime}(z) & \frac{p^{(k-m+1)}(z)}{(k-m+1) !}
\end{array}\right) .
$$

In particular, the expansion formula (3.3) implies that there exists a disk centered at $\theta$ such that for any $z_{0}$ in this disk the sequence of fixed point iteration

$$
z_{k+1}=B_{m}\left(z_{k}\right), \quad k=0,1, \ldots,
$$

is well-defined, and it converges to $\theta$. If $\theta$ is a simple root, the order of convergence is $m$. More specifically,

$$
\lim _{k \rightarrow \infty} \frac{\left(\theta-z_{k+1}\right)}{\left(\theta-z_{k}\right)^{m}}=(-1)^{m} \frac{\widehat{D}_{m-1, m}(\theta)}{p^{\prime}(\theta)^{m-1}} .
$$

Definition 3.2 For a given complex number $w$, the Basic Sequence is defined as

$$
\left\{B_{m}(w), \quad m=2,3, \ldots\right\} .
$$

Theorem $3.3[9,10]$ For any root $\theta$ of $p(z)$ and any $w$ in the Voronoi cell $V(\theta)$, the Basic Family is pointwise convergent to $\theta$, i.e., the corresponding Basic Sequence satisfies

$$
\lim _{m \rightarrow \infty} B_{m}(w)=\theta
$$

Theorem 3.3 establishes the pointwise convergence of the Basic Family on each Voronoi cell. In what follows, we will prove the uniform convergence of the Basic Family, thus describing a more accurate and powerful description for this convergence.

\section{Persistent Neighborhood of a Root and Its Existence}

We first give a definition.

Definition 4.1 Let $\theta$ be a root of $p(z)$ and $V(\theta)$ its Voronoi cell. Given $r>0$ we say that the disk $N_{r}(\theta)=\{z:|z-\theta|<r\}$ is a persistent neighborhood of $\theta$ if

$$
N_{r}(\theta) \subseteq V(\theta)
$$

and for all $m \geq 2$ the Basic Family member $B_{m}(z)$ is a contraction mapping on $N_{r}(\theta)$. Equivalently, for all $m \geq 2$ and for all $z \in N_{r}(\theta),\left|B_{m}^{\prime}(z)\right|<1$, i.e., the sequence of moduli of the derivatives is uniformly and strictly bounded above by 1 . 
Before proving the existence of a persistent neighborhood, note that differentiation of $B_{m}(z)$ gives

$$
B_{m}^{\prime}(z)=1-p^{\prime}(z) \frac{D_{m-2}(z)}{D_{m-1}(z)}-p(z) \frac{D_{m-2}^{\prime}(z) D_{m-1}(z)-D_{m-2}(z) D_{m-1}^{\prime}(z)}{D_{m-1}(z)^{2}} .
$$

From the definition of $D_{m}(z)$ (see (3.1)), it easily follows that $D_{m}(\theta)=p^{\prime}(\theta)^{m}$. Hence when $\theta$ is a simple root,

$$
B_{m}^{\prime}(\theta)=1-p(\theta) \frac{p^{\prime}(\theta)^{m-2}}{p^{\prime}(\theta)^{m-1}}=0 .
$$

This implies that for all $m \geq 2$ the order of convergence of $B_{m}(z)$ is at least quadratic, also proving the existence of a local neighborhood of convergence for each $m \geq 2$. However, to prove persistence we must ensure that the neighborhoods will not shrink to $\theta$ as $m$ approaches infinity. Thus, proving the existence of a persistent neighborhood is a more delicate matter than proving that $\left|B_{m}^{\prime}(\theta)\right|<1$ for all $m$.

The proof of the existence of a persistent neighborhood requires several auxiliary lemmas.

\section{Lemma 4.2 [10] Set}

$$
\Delta_{m}(z)=\left(\begin{array}{ccccc}
p^{\prime} & \sqrt{p} \frac{p^{\prime \prime}}{2 !} & \cdots & \sqrt{p}^{m-2} \frac{p^{(m-1)}}{(m-1) !} & \sqrt{p}^{m-1} \frac{p^{(m)}}{(m) !} \\
\sqrt{p} & p^{\prime} & \ddots & \ddots & \sqrt{p}^{m-2} \frac{p^{(m-1)}}{(m-1) !} \\
0 & \sqrt{p} & \ddots & \ddots & \vdots \\
\vdots & \vdots & \ddots & \ddots & \sqrt{p} \frac{p^{\prime \prime}}{2 !} \\
0 & 0 & \ldots & \sqrt{p} & p^{\prime}
\end{array}\right),
$$

where $\sqrt{p(z)}$ is taken to be the principal value of the complex number $p(z)$. Then

$$
D_{m}(z)=\operatorname{det}\left(\Delta_{m}(z)\right) \text {. }
$$

Lemma 4.3 [10,13] Let $A$ be an $m \times m$ matrix with complex entries. Assume we are given positive lower and upper bounds $l$ and $u$ such that if $\lambda$ is an eigenvalue of $A$ then $l \leq|\lambda| \leq u$. Suppose

$$
|\operatorname{trace}(A)| \geq m \cdot l
$$

Then

$$
|\operatorname{det}(A)| \geq l^{\kappa} u^{m-\kappa},
$$

where

$$
\kappa=\frac{m \cdot u-|\operatorname{trace}(A)|}{u-l} .
$$


Lemma 4.4 Let $\theta$ be a simple root of $p(z)$. There exist positive numbers $r<1, L$, and $\delta<1$ such that for all $z$ in the neighborhood $N_{r}(\theta)$, and all $m \geq 2$ we have

$$
\left|D_{m}(z)\right| \geq\left(\sqrt{1-\delta^{2}} L\right)^{m}
$$

Proof Since $\theta$ is a simple root of $p(z)$, there exists a neighborhood $N_{r^{\prime}}(\theta)$ and a positive constant $L$ such that for all $z \in N_{r}(\theta)$ we have

$$
\left|p^{\prime}(z)\right| \geq L \text {. }
$$

Set

$$
\delta(z)=\frac{1}{\left|p^{\prime}(z)\right|}\left(\sqrt{|p(z)|}+\sum_{i=2}^{n} \frac{\left|p^{(i)}(z)\right|}{i !}|p(z)|^{\frac{i-1}{2}}\right) .
$$

Since $\delta(\theta)=0$, there exists a neighborhood $N_{r^{\prime \prime}}(\theta)$ and a positive constant $\delta$ such that for all $z \in N_{r^{\prime \prime}}(\theta)$ we have

$$
\delta(z) \leq \delta<1 .
$$

Let $r=\min \left\{r^{\prime}, r^{\prime \prime}\right\}$. For each $z \in N_{r}(\theta)$, we have $\delta(z)<1$. This implies that the matrix $\Delta_{m}(z)$ is diagonally dominant. Then, from Gerschgorin's theorem, any eigenvalue $\lambda$ of $\Delta_{m}(z)$ must satisfy the bounds

$$
(1-\delta(z))\left|p^{\prime}(z)\right| \leq \lambda \leq(1+\delta(z))\left|p^{\prime}(z)\right|
$$

Furthermore, we have

$$
\left|\operatorname{trace}\left(\Delta_{m}(z)\right)\right|=m\left|p^{\prime}(z)\right| \geq m(1-\delta)\left|p^{\prime}(z)\right| .
$$

Thus Lemma 4.3 applies to $\Delta_{m}(z)$, where

$$
\kappa=\frac{m(1+\delta(z))\left|p^{\prime}(z)\right|-m\left|p^{\prime}(z)\right|}{(1+\delta(z))\left|p^{\prime}(z)\right|-(1-\delta(z))\left|p^{\prime}(z)\right|}=\frac{m}{2} .
$$

In particular,

$$
\begin{aligned}
\left|D_{m}(z)\right| & \geq\left((1-\delta(z))\left|p^{\prime}(z)\right|\right)^{\frac{m}{2}} \times\left((1+\delta(z))\left|p^{\prime}(z)\right|\right)^{\frac{m}{2}} \\
& =\left(\sqrt{1-\delta^{2}(z)}\left|p^{\prime}(z)\right|\right)^{m} \geq\left(\sqrt{1-\delta^{2}} L\right)^{m} .
\end{aligned}
$$

Hence the proof.

Lemma 4.5 Let $N_{r}(\theta)$ be as in Lemma 4.4. Let $M$ be a number such that for all $z \in N_{r}(\theta)$ we have

$$
\sqrt{\left(\sum_{i=0}^{n} \frac{\left|p^{(i)}(z)\right|}{i !}\right)^{2}} \leq M
$$


Then for each $z \in N_{r}(\theta)$, each $m \geq 1$, and each $k=m+1, \ldots, m+n-1$, we have

$$
\max \left\{\left|D_{m}(z)\right|,\left|\widehat{D}_{m, k}(z)\right|\right\} \leq M^{m} .
$$

Proof First, note that the quantity $M$ bounds the norm of each column of the matrices corresponding to the determinants $D_{m}(z)$ and $\widehat{D}_{m, k}(z)$, see (3.1) and (3.4). From Hadamard's inequality, the modulus of the determinant of a matrix is bounded above by the product of the norm of the columns. Hence the proof.

Lemma 4.6 Let $N_{r}(\theta)$ and $M$ be as in Lemma 4.5. Then for each $z \in N_{r}(\theta)$, and each $m \geq 1$, and each $k=m+1, \ldots, m+n-1$, we have

$$
\max \left\{\left|D_{m}^{\prime}(z)\right|,\left|\widehat{D}_{m, k}^{\prime}(z)\right|\right\} \leq n m M^{m} .
$$

Proof Given an $m \times m$ matrix $A(z)=\left(a_{i j}(z)\right)$ with entries that are analytic functions of $z$, the following formula is known, and easily provable via induction,

$$
\frac{d}{d z} \operatorname{det}(A(z))=\sum_{j=1}^{m} \operatorname{det}\left(\begin{array}{ccccc}
a_{11}(z) & \ldots & a_{1 j}^{\prime}(z) & \ldots & a_{1 m}(z) \\
a_{21}(z) & \ldots & a_{2 j}^{\prime}(z) & \ldots & a_{2 m}(z) \\
\vdots & \vdots & \vdots & \vdots & \\
a_{m 1}(z) & \ldots & a_{m j}^{\prime}(z) & \ldots & a_{m m}(z)
\end{array}\right) .
$$

Applying the above property, it follows that $D_{m}^{\prime}(z)$ is the sum of $m$ determinants each of which corresponds to a matrix where the norm of any column that is not differentiated is bounded above by $M$. After differentiating $D_{m}(z)$, a bound on the norm of each column is given by

$$
\sqrt{\left(\sum_{i=1}^{n} \frac{\left|p^{(i)}(z)\right|}{(i-1) !}\right)^{2}}=\sqrt{\left(\sum_{i=1}^{n} i \frac{\left|p^{(i)}(z)\right|}{i !}\right)^{2}} \leq \sqrt{n^{2}\left(\sum_{i=0}^{n} \frac{\left|p^{(i)}(z)\right|}{i !}\right)^{2}} \leq n M .
$$

Thus from Hadamard's inequality the modulus of the determinant of each of these $m$ matrices is bounded above by $n M^{m}$. This proves the claimed bound on $\left|D_{m}^{\prime}(z)\right|$. Similar argument proves the bound for $\left|\widehat{D}_{m, k}(z)\right|$.

Theorem 4.7 Suppose $\theta$ is a simple root of $p(z)$. Then there exists a persistent neighborhood of $\theta$. More specifically, let the neighborhood $N_{r}(\theta)$ and the constant $M$ be as in Lemma 4.5. Set

$$
\mu=\frac{M}{\sqrt{1-\delta^{2}} L} .
$$

Then for all $z \in N_{r}(\theta)$, we have

$$
\left|B_{m}^{\prime}(z)\right| \leq \frac{n\left(2 \mu+4 \mu^{2}\right)}{1-r}|\theta-z|^{m-1} .
$$

In particular, if we choose $r_{*}<r$ also satisfying

$$
r_{*}<\frac{1-r}{n\left(2 \mu+4 \mu^{2}\right)}
$$


then for all $z \in N_{r_{*}}(\theta)$

$$
\left|B_{m}^{\prime}(z)\right| \leq r_{*}^{m-2}
$$

so that $N_{r_{*}}(\theta)$ is a persistent neighborhood of $\theta$. Moreover, on $N_{r_{*}}(\theta)$ the sequence $B_{m}^{\prime}(z)$ uniformly converges to zero.

Proof To prove (4.2), for each $m \geq 2$ we differentiate the expansion formula for $B_{m}(z)$, see (3.3). Suppressing the variable $z$, we then get

$$
B_{m}^{\prime}(z)=U(z)+V(z)
$$

where

$$
\begin{aligned}
& U(z)=(-1)^{m+1} \sum_{k=m}^{m+n-2} k \frac{\widehat{D}_{m-1, k}}{D_{m-1}}(\theta-z)^{k-1}, \\
& V(z)=(-1)^{m} \sum_{k=m}^{m+n-2} \frac{\widehat{D}_{m-1, k}^{\prime} D_{m-1}-\widehat{D}_{m-1, k} D_{m-1}^{\prime}}{D_{m-1}^{2}}(\theta-z)^{k} .
\end{aligned}
$$

From Lemmas 4.4 and 4.5, we have

$$
\frac{\widehat{D}_{m-1, k}}{D_{m-1}} \leq\left(\frac{M}{\sqrt{1-\delta^{2}} L}\right)^{m-1}=\mu^{m-1} .
$$

Using the above bound, and since $|\theta-z|<r<1$ implies $|\theta-z|^{k} \leq|\theta-z|^{k-1}$, together with the fact that $k \leq(m+n-2) \leq n 2^{m-1}$, we have

$$
\begin{aligned}
|U(z)| & \leq(m+n-2)(\mu|\theta-z|)^{m-1} \sum_{i=0}^{\infty}|\theta-z|^{i} \\
& =(m+n-2)(\mu|\theta-z|)^{m-1} \frac{1}{1-|\theta-z|} \\
& \leq \frac{n}{1-r}(2 \mu|\theta-z|)^{m-1} .
\end{aligned}
$$

Similarly, from Lemmas 4.4, 4.5, 4.6 and since $(2 m-2)<2^{2 m-2}$, we have

$$
\begin{aligned}
\frac{\left|\widehat{D}_{m-1, k}^{\prime} D_{m-1}-\widehat{D}_{m-1, k} D_{m-1}^{\prime}\right|}{\left|D_{m-1}^{2}\right|} & \leq \frac{\left|\widehat{D}_{m-1, k}^{\prime}\right|\left|D_{m-1}\right|+\left|\widehat{D}_{m-1, k}\right|\left|D_{m-1}^{\prime}\right|}{\left|D_{m-1}\right|^{2}} \\
& \leq\left(\frac{2 n(m-1) M^{2 m-2}}{\left(\sqrt{1-\delta^{2}} L\right)^{2 m-2}}\right) \\
& \leq n\left(\frac{2 M}{\sqrt{1-\delta^{2}} L}\right)^{2 m-2}=n(2 \mu)^{2 m-2} .
\end{aligned}
$$


Using the above bound and since $|\theta-z|^{m} \leq|\theta-z|^{m-1}$, we get

$$
|V(z)| \leq n(2 \mu)^{2 m-2}|\theta-z|^{m} \sum_{i=0}^{\infty}|\theta-z|^{i} \leq \frac{n}{1-r}\left(4 \mu^{2}|\theta-z|\right)^{m-1} .
$$

The proof of (4.2) follows from the derived bounds on $|U(z)|$ and $|V(z)|$, and the observation that

$$
(2 \mu)^{m-1}+\left(4 \mu^{2}\right)^{m-1} \leq\left(2 \mu+4 \mu^{2}\right)^{m-1} .
$$

From (4.2) the remaining claimed bounds are trivially derivable.

Remark 4.8 Note that Theorem 4.7 establishes the uniform convergence of the sequence of $B_{m}^{\prime}(z)$ to zero on a neighborhood of $\theta$. This is a much stronger result than proving the existence of a neighborhood of $\theta$ with $\left|B_{m}^{\prime}(z)\right|<1$. While we have assumed the simplicity of the root $\theta$, the existence of persistent neighborhood can be extended to the case where $\theta$ is a multiple root. Although $D_{m}(\theta)$ is zero at a multiple root, the expansion in (3.3) remains valid and $B_{m}(z)$ can be defined at such multiple root. This is the case, for example, for $B_{2}(z)=z-p(z) / p^{\prime}(z)$, namely Newton's iteration function. In such a case, $B_{m}^{\prime}(\theta)$ is nonzero, nevertheless $\left|B_{m}^{\prime}(\theta)\right|<1$ for all $m \geq 2$. More specifically, if $\theta$ is a root of multiplicity $s$, it can be shown that $B_{m}^{\prime}(\theta)=(s-1) /(s+m-2)$, see [10], Theorem 6.1, or [12]. We avoid details.

\section{Approximation of Voronoi Diagram}

We are finally ready to prove our main theorem.

Theorem 5.1 Suppose that $\theta$ is a simple root of $p(z)$ with at least three distinct roots. Let $C$ be any closed set contained in $V(\theta)$. Then there exists $m_{0}$ such that for all $m \geq m_{0}, A_{m}(\theta)$, the basin of attraction of $\theta$ with respect to $B_{m}(z)$, contains $C$.

Proof We consider two cases.

Case I. Assume $V(\theta)$ is a bounded set. Let $N_{r}(\theta)$ be a persistent neighborhood of $\theta$. From Theorem 3.3, for each $w \in C$ there exists a natural number $m_{w}$ such that for all $m \geq m_{w}, B_{m_{w}}(z)$ lies in $N_{r}(\theta)$. In particular,

$$
O_{w}=B_{m_{w}}^{-1}\left(N_{r}(\theta)\right)
$$

is an open set containing $w$. This implies the family $\left\{O_{w}: w \in C\right\}$ is an open cover for $C$. Since $C$ is closed and bounded (as $V(\theta)$ is bounded), by compactness there exists a finite subcover for $C$ :

$$
C \subseteq \bigcup_{i=1}^{t} O_{w_{t}}
$$

Let $m_{0}=\max \left\{m_{w_{1}}, \ldots, m_{w_{t}}\right\}$. It follows that for each $z \in C$ and each $m \geq m_{0}$ 
we have

$$
B_{m}(z) \subseteq A_{m}(\theta) .
$$

This completes the proof for the case where $V(\theta)$ is bounded.

Case II. Assume $V(\theta)$ is unbounded. Consider the stereographic projection of the complex plane into the Riemann sphere and the topology induced by the chordal metric defined for two complex numbers $z$ and $w$ as

$$
\sigma(z, w)=\frac{2|z-w|}{\sqrt{\left(1+|z|^{2}\right)\left(1+|w|^{2}\right)}},
$$

see, e.g., [3].

Let $\widehat{\theta}$ be the stereographic projection of $\theta$, and $\widehat{V}(\widehat{\theta})$ the stereographic projection of $V(\theta)$. Let $\widehat{C}$ be the stereographic projection of $C$. Note that $\widehat{C}$ is closed and bounded, hence compact. Under the stereographic projection, the persistent neighborhood $N(\theta)$ gets mapped into a persistent neighborhood $\widehat{N}_{r}(\widehat{\theta})$ of $\widehat{\theta}$. For all $m \geq 2$, the point $\infty$ can be shown to be a repelling fixed point of $B_{m}(z)$ (see [10], Theorem 5.3) so that, under stereographic projection, $\infty$ gets mapped to a boundary point of $\widehat{V}\left(\widehat{\theta}^{\prime}\right)$, for some root $\theta^{\prime}$ of $p(z)$.

Analogous to Case I, we use the boundedness of $\widehat{V}(\widehat{\theta})$, the persistence of $\widehat{N}_{r}(\widehat{\theta})$, and the compactness of $\widehat{C}$, in order to draw the desired conclusion.

Next we state a corollary of the above result according to which for each given polynomial with simple roots, the Basic Family members uniformly approximate Voronoi cells of its roots with their corresponding basins of attraction. In particular, the chaotic behavior of iterations of $B_{m}(z)$ can be controlled to uniformly lie to within a strip neighborhood of the boundary of the Voronoi diagram.

Corollary 5.2 Let $p(z)$ be a complex polynomial with at least three distinct roots. Assume all roots of $p(z)$ are simple. Let $G$ denote the graph consisting of the vertices and edges of the Voronoi cell of the roots (e.g., the skeleton in Fig. 1). Given $\epsilon>0$, let $G_{\epsilon}$ correspond to an inflated $G$ where each line in $G$ is inflated to an open strip of width $\epsilon$. Let $V_{\epsilon}$ be the complement of $G_{\epsilon}$. Then there exists $m_{\epsilon}$ such that for all $m \geq m_{\epsilon}$, the basins of attraction of $B_{m}(z)$ contains $V_{\epsilon}$. In particular, if $J_{m}$ denotes the Julia set of $B_{m}(z)$, then for all $m \geq m_{\epsilon}, G_{\epsilon}$ contains $J_{m}$.

It would be interesting and perhaps nontrivial to determine the dependence of $m_{\epsilon}$ on $\epsilon$ and the coefficients of the given polynomial $p(z)$.

\section{Layering of Points in Voronoi Cells}

In this section, we will make use of the Basic Sequence (see Definition 3.2) to define a layering of the points within a given Voronoi cell. Before describing the approach, we offer a motivation. Consider a given set of points in the Euclidean plane:

$$
S=\left\{p_{i}=\left(x_{i}, y_{i}\right), i=1, \ldots, n\right\},
$$


and assume that we have computed their Voronoi diagram

$$
\left\{V\left(p_{1}\right), \ldots, V\left(p_{n}\right)\right\},
$$

where for each $i=1, \ldots, n, V\left(p_{i}\right)$ denotes the Voronoi cell of $p_{i}$. To measure the relative merit of points in a given Voronoi cell $V\left(p_{j}\right)$, we need to define a meaningful hierarchy. An intuitive hierarchy would be to measure their Euclidean distance to $p_{j}$. However, this completely ignores the relationship of the points inside the cell, to the points outside of the cell and the way in which they have influenced the formation of the cell to begin with. In what follows, we will introduce a notion of distance on points in a given Voronoi cell. This notion uses the encoding of the points as a polynomial equation.

Consider each point $p_{j}=\left(x_{j}, y_{j}\right)$ as a complex number

$$
\theta_{j}=x_{j}+\mathbf{i} y_{j}, \quad \mathbf{i}=\sqrt{-1} .
$$

Set $p(z)$ to be the polynomial having all $\theta_{j}$ 's as its roots, and only these points:

$$
p(z)=\prod_{i=1}^{n}\left(z-\theta_{i}\right)=\sum_{i=0}^{n} a_{i} z^{i}
$$

for some set of complex coefficients $a_{i}, i=0, \ldots, n$. If we assume that the input to a Voronoi diagram algorithm is the set of coefficients, as opposed to the set of coordinates, the problem of computing the Voronoi diagram now completely differs from its ordinary version.

Our approach in this section is to consider the Voronoi diagram of a set of points in the Euclidean plane using the fact that the points can be described via a polynomial equation. This polynomial could be given explicitly in terms of its coefficients, or explicitly in terms of the roots. We will explore the relation between Voronoi diagram to polynomial root-finding via the Basic Family.

Definition 6.1 Let $p(z)$ be a polynomial of degree $n$. Let $\theta$ be a root of $p(z)$. Assume that $N_{r}(\theta)$ is a neighborhood contained in $V(\theta)$. Given a point $z \in V(\theta)$, the layer of $z$ is denoted by $l_{r}(z)$ and defined as follows:

$$
l_{r}(z)= \begin{cases}0, & \text { if } z \in N_{r}(\theta) \\ \min \left\{m-1: B_{m}(z) \in N_{r}(\theta), m \geq 2\right\}, & \text { if } z \in V(\theta)-N_{r}(\theta) \\ \infty, & \text { if } z \notin V(\theta) .\end{cases}
$$

From Theorem 3.3, the function $l_{r}(z)$ is well-defined for all complex numbers $z$, whether or not $\theta$ is a simple root. This gives rise to a pseudometric $d(\cdot, \cdot)$ on points $u$ and $v$ in the Euclidean plane

$$
d(u, v)=\left|l_{r}(u)-l_{r}(v)\right| .
$$

Definition 6.2 A layer of depth $m$ with respect to $\theta$, denoted by $L_{m}$, is the set of all $z$ that are at layer $m$, i.e.,

$$
L_{m}=\left\{z: l_{r}(z)=m\right\}
$$


Theorem 6.3 Let $p(z)$ be a complex polynomial having at least three distinct roots. Let $\theta$ be a root of $p(z)$. Let $r>0$ be such that $N_{r}(\theta)$ is a persistent neighborhood of $\theta$. Let $l_{r}(z)$ be the corresponding layering. Then $V(\theta)$ contains layers of all depths, i.e., for all $m \geq 1$, there exists $z \in V(\theta)$ such that $l_{r}(z)=m$.

Proof If there are only finitely many layers, then there exists $m_{0} \geq 2$ such that for all $z_{0} \in V(\theta)$,

$$
B_{m_{0}}\left(z_{0}\right) \in N_{r}(\theta) .
$$

This together with the assumption that $N_{r}(\theta)$ is a persistent neighborhood of $\theta$ implies that the orbit of $z_{0}$ with respect to $B_{m_{0}}(z)$ converges to $\theta$. Thus, $V(\theta)$ is contained in $A_{m_{0}}(\theta)$, the basin of attraction of $\theta$ with respect to $B_{m_{0}}$. We may thus conclude:

$$
V(\theta) \subseteq B_{m_{0}}^{-1}\left(N_{r}(\theta)\right)
$$

We claim the above containment cannot be strict so that we must have

$$
V(\theta)=B_{m_{0}}^{-1}\left(N_{r}(\theta)\right)
$$

Suppose this equality is not valid. Since $B_{m_{0}}^{-1}\left(N_{r}(\theta)\right)$ is the inverse image of an open set, under continuity of $B_{m_{0}}$ it must be an open set itself. Therefore, there exists $z_{0} \in V\left(\theta^{\prime}\right)$, where $\theta^{\prime}$ is some other root of $p(z)$. But, from Theorem 3.3, this implies that the Basic Sequence $\left\{B_{m}\left(z_{0}\right)\right\}_{m=m_{0}}^{\infty}$ converges to $\theta^{\prime}$. This contradicts the fact that $N_{r}(\theta)$ is contained in $A_{m_{0}}(\theta)$. Hence the proof of the claim. It follows that we must have

$$
V(\theta)=A_{m_{0}}(\theta) .
$$

But, by Theorem 2.2, this is a contradiction. Hence the proof.

Next we prove a more general version of the above theorem where $N_{r}(\theta)$ is an arbitrary neighborhood of $\theta$ contained in $V(\theta)$. This settles a question raised in our earlier work [11].

Theorem 6.4 Let $p(z)$ be a complex polynomial having at least three distinct roots. Let $\theta$ be a simple root of $p(z)$. Let $N_{r}(\theta)$ be any neighborhood of $\theta$ contained in $V(\theta)$. Let $l_{r}(z)$ be a layering defined with respect to $N_{r}(\theta)$. Then, $V(\theta)$ contains layers of all depths.

Proof Let $C$ be the closure of $N_{r}(\theta)$. Then, from Theorem 5.1 for all $m \geq m_{0}, A_{m}(\theta)$ contains $C$. If $N_{r}(\theta)$ is not already a persistent neighborhood of $\theta$, then from Theorem 4.7 it follows that it must necessarily contain a persistent neighborhood, say $N_{\rho}(\theta)$ for some $0<\rho<r$. Then, from Theorem 6.4, there are layers of all depth with respect to $N_{\rho}(\theta)$. It follows that there are layers of all depth with respect to $N_{r}(\theta)$.

Remark 6.5 In the case where $p(z)$ is a quadratic polynomial, the infinity of layers is still valid. The proof, however, follows from a different argument. In this case for 
each $m \geq 2$, the Julia set of $B_{m}(z)$ is merely the boundary of the two Voronoi cells. This special case is quite interesting in its own right, see Chap. 1 in [10].

Remark 6.6 Voronoi cell layering remains well-defined under multiplicity of the roots. Suppose $p(z)$ is a polynomial with roots $\theta_{i}$ repeated with multiplicity $m_{i}$, $i=1, \ldots, n$. Clearly, the Voronoi cells remain unchanged, but the layering may differ from the case of simple roots. In this case the underlying polynomial is

$$
p(z)=\left(z-\theta_{1}\right)^{m_{1}} \times \cdots \times\left(z-\theta_{n}\right)^{m_{n}}
$$

The corresponding Basic Sequence will still converge for points within each Voronoi cell. However, the distance, $d(u, v)$, would be dependent on the multiplicities.

\subsection{Localization of Layering Using Adjacent Cells}

The layering defined previously needs to make use of the entire set of points in $S=$ $\left\{p_{1}, \ldots, p_{n}\right\}$, hence dealing with a polynomial of degree $n$ which may be prohibitive when $n$ is large. In the remaining of the section, we describe a layering using local information, hence more efficiently computable.

It is well known that the Voronoi cell of a point $p_{j}=\left(x_{j}, y_{j}\right)$ is dependent only on the neighboring Voronoi cells. Furthermore, the average number of neighboring cells is 6, see, e.g., [2]. This suggests an efficient strategy for layering of Voronoi cells of a set of points, when given explicitly through their coordinates. To define the layering, we make use of a local polynomial based on identifying the point $p_{j}$ with the complex number $\theta_{j}=x_{j}+\mathbf{i} y_{j}$. Letting $I_{j}$ be the index set of neighboring points to $p_{j}$, we define the local Voronoi polynomial as

$$
p_{j}(z)=\left(z-\theta_{j}\right) \times \prod_{i \in I_{j}}\left(z-\theta_{i}\right) .
$$

All the stated results regarding layering of the Voronoi cell $V\left(\theta_{j}\right)$ with respect to $p(z)$ are still valid when the layering of the Voronoi cell $V\left(\theta_{j}\right)$ is done with respect to the local polynomial $p_{j}(z)$. This has the advantage that the average degree of the polynomial $p_{j}(z)$ equals 7 . Given a set of $n$ points through their coordinates, we can associate a polynomial to their Voronoi diagram, we call this the Voronoi polynomial:

$$
P(z)=\prod_{j=1}^{n} p_{j}(z)
$$

Voronoi polynomial encodes the Voronoi diagram of the set of points. In future work, we will investigate properties of Voronoi polynomial and the corresponding local layerings. In particular, we will explore this in the context of large-scale polynomiography, a field of study based on the visualization of the process of solving polynomial equations, having potential applications in subject areas that include art, science and education, see [10]. 
Fig. 3 A layering of Voronoi cell of points. The white boundaries correspond to points with layer numbers larger than a certain threshold

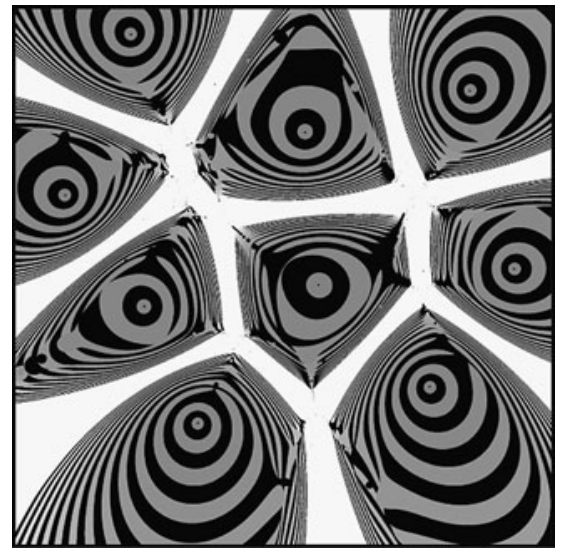

Final Remarks We would like to mention a peculiar connection and a potential novel application of our results. The notion of a zone diagram of a finite set of points in the Euclidean plane is an interesting and rich variation of the classical Voronoi diagram, introduced by Asano, Matoušek, Tokuyama [1]. Certain features in polynomiographs, such as the one given in Fig. 3, resemble images derived from zone diagrams. Indeed, in [5] we have defined a more inclusive notion of a maximal zone diagram (subsequently changed with an improved terminology in [6] as mollified zone diagram). Although the development of maximal zone diagram is independent of our results on polynomial root-finding, the present article suggests as a possibility, approximation of the zone diagram via approximate Voronoi cells based on the use of the Basic Sequence (see Definition 3.2), followed by iterations toward a maximal (mollified) zone diagram.

The proof of existence of maximal zone diagrams depends on less restrictive initial conditions and is thus conveniently established via Zorn's lemma in contrast to the use of fixed-point theory in proving the existence of a unique zone diagram. A zone diagram is a particular maximal zone diagram satisfying a unique dominance property. In [5], we give a characterization for maximal zone diagrams which allows recognition of maximality of certain subsets, called subzone diagram (territory diagram in [6]), as well as that of their iterative improvement toward maximality. Maximal zone diagrams offer their own interesting theoretical and computational challenges. In future work, we hope to explore possible connections between our theory and algorithms for polynomial root-finding, and those of zone diagram, as well as maximal zone diagrams.

\section{References}

1. Asano, T., Matoušek, J., Tokuyama, T.: Zone diagrams: existence, uniqueness, and algorithmic challenge. SIAM J. Comput. 37, 1182-1198 (2007)

2. Aurenhammer, F.: Voronoi diagrams - a survey of fundamental geometric data structure. ACM Comput. Surv. 23, 345-405 (1991)

3. Beardon, A.F.: Iteration of Rational Functions: Complex Analytic Dynamical Systems. Springer, New York (1991) 
4. Cayley, A.: The Newton-Fourier imaginary problem. Am. J. Math. 2, 97 (1879)

5. de Biasi, S.C., Kalantari, B., Kalantari, I.: Maximal zone diagrams and their computation. In: Proceedings of the Seventh Annual International Symposium on Voronoi Diagrams in Science and Engineering, pp. 171-180 (2010)

6. de Biasi, S.C., Kalantari, B., Kalantari, I.: Mollified zone diagrams and their computation (2011, in preparation)

7. Goodman, J.E., O'Rourke, J. (eds.): Handbook of Discrete and Computational Geometry, 2nd edn. Discrete Mathematics and Its Applications. Chapman \& Hall, Boca Raton (2004)

8. Kalantari, B.: Generalization of Taylor's theorem and Newton's method via a new family of determinantal interpolation formulas and its applications. J. Comput. Appl. Math. 126, 287-318 (2000)

9. Kalantari, B.: On homogeneous linear recurrence relations and approximation of zeros of complex polynomials. In: DIMACS Series in Discrete Mathematics and Theoretical Computer Science. Unusual Applications in Number Theory, vol. 64, pp. 125-143 (2004)

10. Kalantari, B.: Polynomial Root-Finding and Polynomiography. World Scientific, New Jersey (2008)

11. Kalantari, B.: Voronoi diagrams and polynomial root-finding. In: Proceedings of the Sixth Annual International Symposium on Voronoi Diagrams in Science and Engineering, pp. 31-40 (2009)

12. Kalantari, B., Jin, Y.: On extraneous fixed-points of the basic family of iteration functions. BIT 43, 453-458 (2003)

13. Kalantari, B., Pate, T.H.: A Determinantal lower bound. Linear Algebra Appl. 326, 151-159 (2001)

14. McMullen, C.: Families of rational maps and iterative root-finding algorithms. Ann. Math. 125, 467493 (1987)

15. Milnor, J.: Dynamics in One Complex Variable: Introductory Lectures, vol. 160, 3rd edn. Princeton University Press, New Jersey (2006)

16. Preparata, F.P., Shamos, M.I.: Computational Geometry: An Introduction. Springer, New York (1985)

17. Schröder, E.: On infinitely many algorithms for solving equations. Math. Ann. 2, 317-365 (1870) (German). (English translation by G.W. Stewart, TR-92-121, Institute for Advanced Computer Studies, University of Maryland, College Park, MD, 1992.)

18. Traub, J.F.: Iterative Methods for the Solution of Equations. Prentice Hall, Englewood Cliffs (1964) 